\title{
The Use of a Modelling \& Simulation Tier by the EMULSION IoT Platform
}

\author{
IVAN GANCHEV \\ Department of Computer Systems, \\ University of Plovdiv "Paisii Hilendarski", \\ 24 Tsar Assen St., Plovdiv 4000, \\ BULGARIA. \\ $\&$ \\ Institute of Mathematics and Informatics, \\ Bulgarian Academy of Sciences, \\ Akad. G. Bonchev St., Block 8, Sofia 1113, BULGARIA. \\ $\&$ \\ Telecommunications Research Centre (TRC), \\ University of Limerick, \\ Plassey, National Technological Park, Co. Limerick, \\ IRELAND. \\ https://orcid.org/0000-0003-0535-7087

\section{ZHANLIN JI} \\ College of Artificial Intelligence, \\ North China University of Science and Technology, \\ Caofeidian, Tangshan City, \\ CHINA. \\ https://orcid.org/0000-0003-3527-3773
}

\begin{abstract}
This paper presents some design aspects of the EMULSION IoT platform, developed as a typical example of the horizontal IoT platforms. The architectural overview and multi-tiered structure of the platform are described, with special attention being paid to its modelling \& simulation tier as a novel architectural element proposed for inclusion in similar IoT platforms. Used to model cyber-physical-social (CPS) objects and IoT services, along with their attributes and temporal/spatial/event characteristics, this tier is also utilized to simulate the actual provision of IoT services in order to determine the optimal configuration of the platform in each particular use case, by solving complex optimization tasks. Examples of such tasks are presented in the paper along with some results obtained to date.
\end{abstract}

Keywords: - IoT platform, horizontal-type, tiers, modelling, simulation.

Received: April 17, 2021. Revised: January 26, 2022. Accepted: February 18, 2022. Published: March 3, 2022.

\section{Introduction}

The provision of Internet of Things (IoT) services requires the use of a proper IoT platform ${ }^{1}$ for ensuring interconnection and interoperability of different types of IoT things, objects, devices, networks, hardware modules, software components, etc., and providing the required functionalities for telco-, device-, data-, application management, etc.

\footnotetext{
${ }^{1}$ IoT platform - a type of a digital platform, used for building and managing IoT solutions [1].
}

[2], with some freedom of use by consumers (along with the required and customization and personalization) [3], which in the cloud domain is known as Platform as a Service (PaaS). IoT platforms play an important role and are of major importance for the entire IoT ecosystem [2]. All companies considering the realization of potential IoT scenarios and use cases should seriously consider the use of a suitable IoT platform that can provide a stable, secure, and flexible solution meeting today's architectural and business requirements [1]. 
The objective of this paper is to point out to the need of using a dedicated modelling \& simulation tier in the IoT platforms for: (i) modelling the utilized cyber-physical-social (CPS) objects and IoT services, along with their attributes and temporal/spatial/event characteristics; and (ii) simulating the actual provision of IoT services as to determine the optimal configuration of the corresponding platform in each particular use case, by solving complex optimization tasks.

The use of a modelling \& simulation tier is demonstrated here on the example of EMULSION [4] - a generic multi-service cloud-based $\underline{\text { IoT }}$ operational platform, which could be used as an architectural foundation for creating 'smart city' IoT systems [5] and for fast roll-out of related IoT services of different types.

\section{State of the Art in the Area of IoT Platforms Development}

Even though in the last two years there was no increase in the number of IoT platform companies, the size of the IoT platforms market increased substantially and it is expected to grow from $\$ 5$ billion in 2020 to $\$ 28$ billion in 2026, which is a $33 \%$ compound annual growth rate (CAGR), as shown in [2]. This growth seems driven by the fast adoption of cloud-based IoT platforms and the rising number of customers who chose to buy an IoT platform rather than build their own. The same source identifies 613 IoT platform providers operating on the market at the end of 2021. The dynamics of the current situation is also recognized there by pointing out that, starting from 2019, many of these platform providers have pivoted their business models - either by no longer focusing on IoT alone and offering a collection of modular, preintegrated artificial intelligence (AI) services (24\% of the IoT platform providers), or having moved toward the sale of IoT application solutions (21\%), or even stopping to exist (26\%). While the number of IoT platform vendors in all other regions of the world decreased during the 2019-2021 period, in the Asia-Pacific region, and especially in China, the number of IoT platforms increased strongly. In total, 182 new IoT platform companies emerged since 2019, and 66 of these operate in China [2].

Commercial IoT platforms could be classified into five general types [2]:

- Telco management/connectivity platforms (constituting 7\% of the 613 IoT platforms existed by the end of 2021, [2]) - used to manage the connectivity and control the traffic to/from IoT devices at scale, primarily through $2 \mathrm{G} \div 6 \mathrm{G}$ cellular networks, but also via Low-Power Wide Area Networks (LPWANs) such as Sigfox and LoRa, and potentially fixed and satellite connections. Typical functionalities include billing management, connectivity orchestration, connectivity management, and service provisioning [1]. As part of the subscription, add-ons may also be included, e.g., bill analyzers, usage anomaly detectors, etc. Many cellular operators are relying on this type of IoT platforms (and probably will continue to do so) to scale up their IoT deployments and extend their services to new verticals [6]. In addition, top IoT platform vendors are continuing to support multiple use cases across different verticals [7]. Examples of this type of IoT platforms include Cisco's Jasper, Ericsson's DCP, Huawei Connection Management Platform, Verizon's network + ThingSpace, etc.

- Device management/enablement platforms (constituting 35\%, [2]) - providing an ability to remotely configure, monitor, control, and manage IoT devices. Functionalities of these include over-the-air firmware updates, deployment configuration, device monitoring, command and control, etc. [1].

- Data management platforms (constituting $43 \%$ of the 613 IoT platforms existed by the end of 2021, [2]) - providing an ability to ingest, store, and analyze data from IoT devices. Typical functionalities include data storage support (data bases, data lakes, data warehouses), data analysis (rules engines / event management, data preparation, extraction, transformation, load - ETL, data analytics, AI / machine learning), and southbound data ingest/egress (data acquisition drivers and interfaces, IoT hubs, IoT device software development kits, data brokers) [1].

- Application management/enablement platforms (constituting 58\% of the 613 IoT platforms existed by the end of 2021, [2]) providing an ability to rapidly develop, test, verify, validate, and manage IoT applications of different types. Standard functionalities include application management (IoT application marketplace and application lifecycle management), application development (digital twins, integrated development environments - IDEs), northbound data ingest/egress (application 
programming interface (API) management, alert/notification services) [1]. IoT platform vendors, as well as some third parties, monetize applications built on top of the IoT platforms of this type. Typical examples include the Siemens' Closed-Loop Foundation application for MindSphere and the Edge2Web's Director application for MindSphere, [1].

- IoT-based Infrastructure-as-a-Service (IaaS) - these constitute only $3 \%^{2}$ of the 613 IoT platforms existed by the end of 2021, according to [2]. However, cloud hyperscalers (e.g., Microsoft, AWS, Google) realize IaaS revenue also by hosting the IoT platforms of other companies on their infrastructures. Examples include MachineMetrics on AWS IoT Core, Uptake on Microsoft's Azure, and Oden Technologies on Google Cloud, [1].

Most IoT platform companies today are now offering vertical solutions alongside their platforms [2]. This contrasts with the horizontal design principles for achieving easy integration and full interoperability that holds much potential for creating new business opportunities and associated IoT services, and for eliminating the duplicate solutions, thus simplifying the existing IoT environments. Equipped with this new approach, each service, application, or network provider can supply a complete horizontal-slice solution, applicable to multiple IoT domains, with greater possibility to easy and timely adjust its operation to new emerging IoT scenarios and use cases, and with efficient operation, administration, and management (OAM) of the IoT ecosystem throughout the entire lifetime.

For non-hyperscaler IoT platform vendors, it is extremely difficult nowadays to compete with cloud hyperscalers, which capture an increasing quantity of revenue with respect to platforms (via PaaS) and/or computing infrastructures (via IaaS), [1]. So, non-hyperscaler IoT platform vendors are progressively focusing on more vertical purposebuilt specific applications (e.g., GE Digital), services (e.g., Accenture), or solutions (e.g., Siemens), [1]. As the platform layer itself becomes less differentiated, many companies seem increasingly offering more vertical or use-case specific solutions (both hardware and software) by leveraging some underlying IoT platform, [1]. Large multinationals and big enterprises have selected at

\footnotetext{
2 The total number of percentages is greater than $100 \%$ because some of the platforms are identified in [2] as being of more than one type.
}

early stage the IoT platform(s) for their own usage. For instance, Walmart selected a single IoT platform (Azure) for use, but Volkswagen selected several IoT platforms (Siemens, AWS, and Azure), [1]. However, most small and medium enterprises (SMEs) cannot afford the use of big-vendor platforms. Following the horizontal trend, EMULSION is developed as a horizontal IoT platform of a combined (hardware and software) type as to satisfy the needs of SMEs.

\section{EMULSION IoT Platform}

Building a robust and reliable, but at the same time low-cost, IoT platform is a difficult task, taking into account the fact that each such platform comprises multiple heterogeneous hardware elements (e.g., semiconductors, sensors, actuators, monitoring stations, controllers, guards, single-board computers, communication modules, etc.) and software components (e.g., embedded operating systems, distributed message queues, producerconsumer subsystems, machine-learning APIs, applications, tools, utilities, databases, dews/fogs/clouds, etc.). With millions of repositories of open-source software components and hardware modules available on GitHub ${ }^{3}$, choosing the right hardware elements and software code in numerous technical routes is a big research and development (R\&D) challenge.

EMULSION is being built by means of low-cost electronic modules and open-source software components, by utilizing the multi-tier IoT architecture shown in Figure 1 and Figure 2. The sensors (S), location trackers (T) [8], and monitoring stations (MS), deployed in the sensor tier for detecting and reporting changes occurring in the physical world, communicate with the information centers, located in the cloud tier, by means of data/remote transfer units (D/RTUs) [9], which support different wireless communication standards for $2 \mathrm{G} \div 6 \mathrm{G}$ cellular, LoRaWAN, WLAN (Wi-Fi), Bluetooth Low Energy (BLE), etc., communications, and through smart gateways, which ensure the required interoperability between the involved heterogeneous IoT things, objects, devices, nodes, etc. Additionally, wireless sensor networks (WSNs) are established, where needed, for extending the communication range and reaching the corresponding communication gateway(s). This way the information centers in the cloud tier can collect and analyze the data coming from the sensor

\footnotetext{
${ }^{3}$ https://github.com/
} 
tier as to come up with appropriate decisions and recommendations that are sent back as a configuration information and/or commands to the respective actuators (A), controllers (C), and guards
(G), this way enforcing the necessary OAM actions in the sensor tier and realizing the desired/required changes in the physical world.

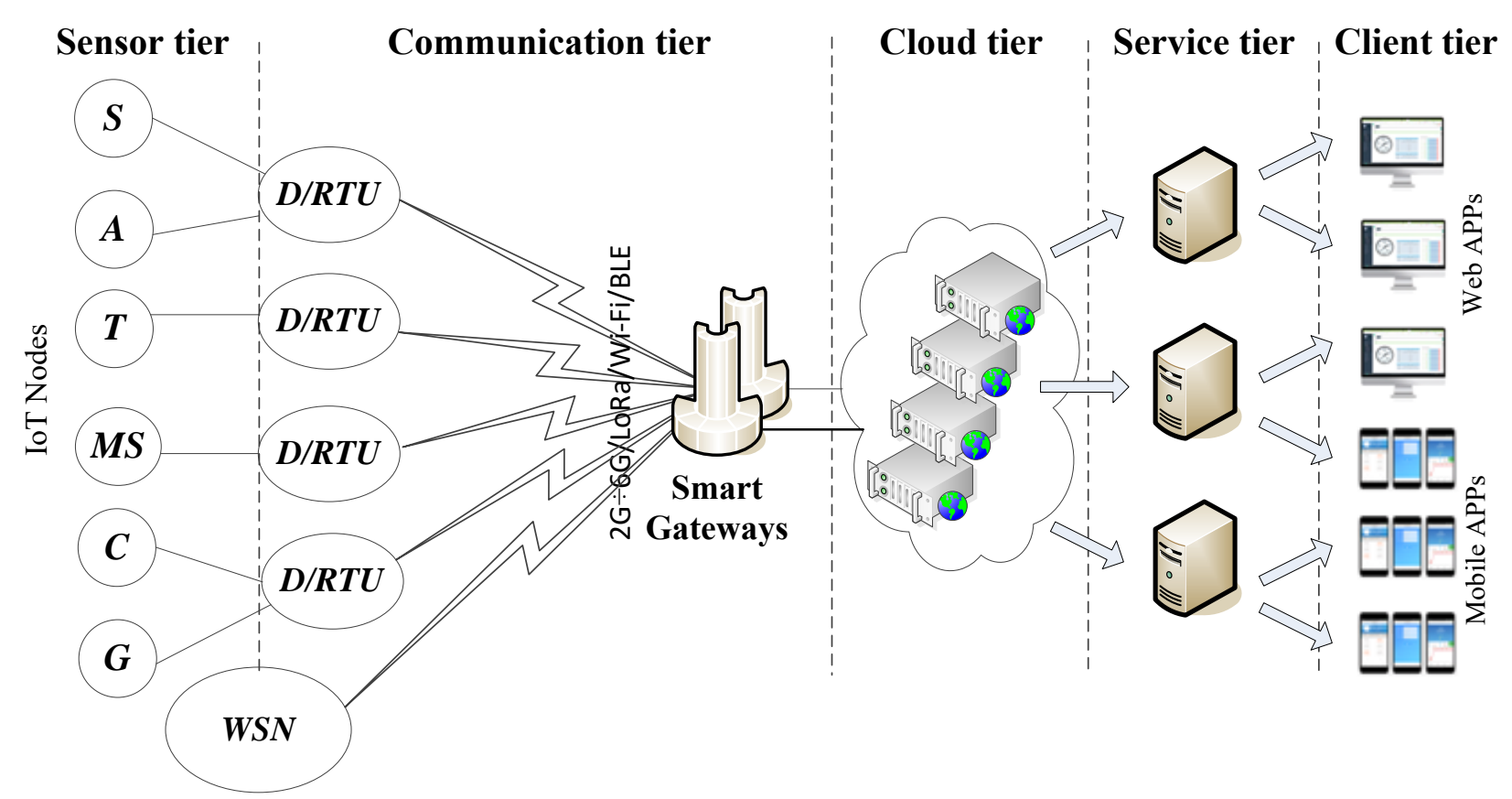

Figure 1. The IoT multi-tier architecture of EMULSION.

EMULSION comprises seven tiers, which are depicted in Figure 2 and briefly described below:

1) Sensor tier - encompassing two groups of IoT nodes: (i) sensors, monitoring stations, location trackers [8], etc., for detecting and notifying about the changes happening in the physical world; and (ii) actuators, controllers, guards, etc., for imposing required changes in the physical world.

2) Communication tier - comprising a variety of D/RTUs [9] and smart communication gateways, facilitating communication between IoT nodes in the sensor tier, accessible via different types of communication networks, and information centers in the cloud tier, and ensuring the required interoperability.

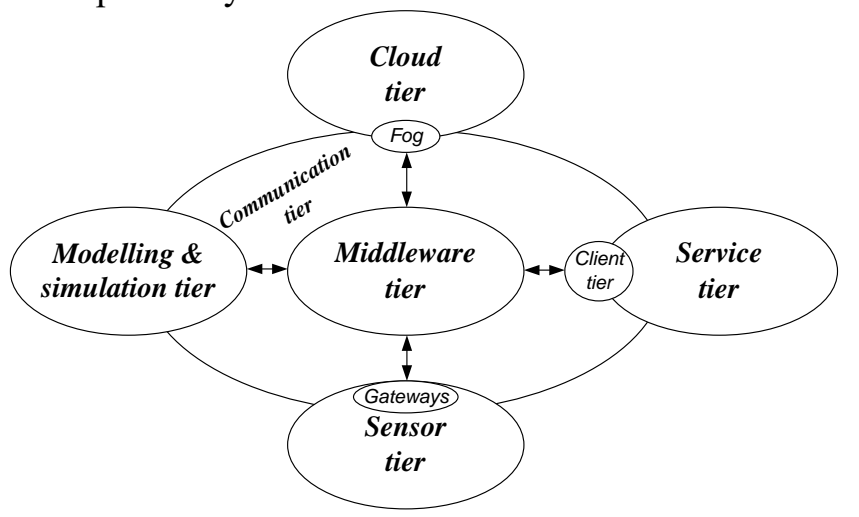

Figure 2. The 7-tier structure of EMULSION.
3) Modelling \& simulation tier - used for: (i) modelling of CPS objects and IoT services (and their inherent attributes and temporal/spatial/event characteristics); and (ii) simulating the actual provision of IoT services. This is a new type of tier, proposed here for inclusion in the IoT platforms. More details about this are provided in the next section.

4) Client tier - containing different smart clients and personal assistant applications, this tier facilitates the consumers' access to the services supplied by the platform, by considering all the current context in order to use the 'best' service instances at any given moment, in conformance to the Always Best Connected and best Served (ABC\&S) communication paradigm [10].

5) Service tier - providing two main types of services: (i) regular IoT services, mostly intended for the 'smart environment control' and 'smart health' [11] [12] IoT domains. Each such service is delivered in a highly personalized, customized, and contextualized ABC\&S way to each particular consumer. The Internet of Services (IoS) [16] is another principle taken on board for facilitating the integration of different (atomic) IoT services into composite services in such a way that allows for their easy discovery, interlinking, mash up, aggregation, and composition into a single service 
bundle; and (ii) supplementary services, used to discover and recommend to each consumer the 'best' instances of the regular IoT services, preferred by him/her. A prototype recommendation service is being developed for consumers to help them plan pro-actively in advance, and change dynamically afterwards if needed, the routes to follow in their movement through poor air-quality/polluted areas, in accordance with the consumers' personal health status. Such a novel health-related criterion (and corresponding service) for route generation and recommendation to consumers could (and should) be included in the existing navigation systems and applications alike. For achieving intelligent service recommendations, suitable models, algorithms, and techniques were elaborated [13] [14] for use by EMULSION.

6) Middleware tier - operating as a medium between all other tiers for ensuring their interoperability [15] and as a central manager of the IoT devices, this tier is concerned also with the proper OAM of the IoT devices and their integration into EMULSION. Moreover, this tier deals with issues related to programmability, scalability, and mapping of the available IoT devices to the supplied IoT services. Applications scheduling is also done by this tier [16].

7) Cloud tier - furnished with a Data Management Platform (DMP) core, this tier deals with 'big data' processing and analytics issues, and converting the raw sensing data gathered from the physical world into actionable analytic datasets that could be properly used by the supplied IoT services. For creating an efficient and effective DMP, the Lambda architecture [17] was selected for utilization (Figure 3).

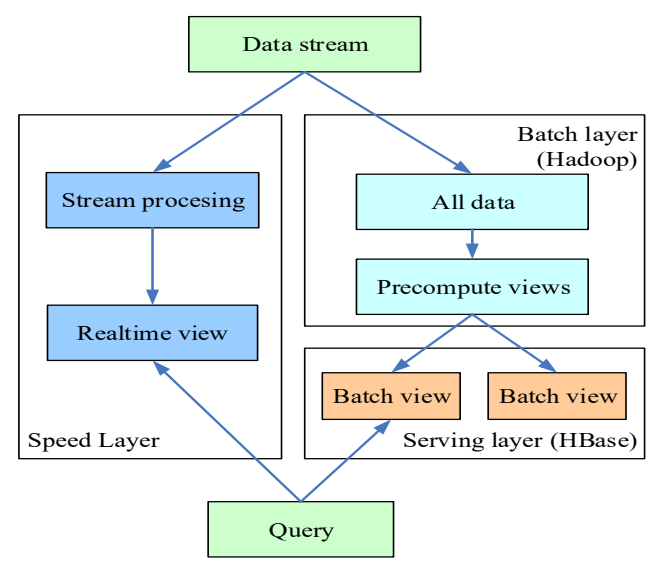

Figure 3. The utilized Lambda architecture.
A distributed, Kafka ${ }^{4}$-based, publish-subscribe messaging module was designed for use in the data stream part for achieving a high-throughput data processing (Figure 4). A second Kafka-based module was designed for the data subscription and archival of messages in the query part, by means of ETL batched consumption operations. For fetching the available topics and storing these on $\mathrm{HDFS}^{5}$, a Camus $^{6}$-based pipeline between Kafka and HDFS is used. For the DMP functions encapsulation, a shared interface class was elaborated.

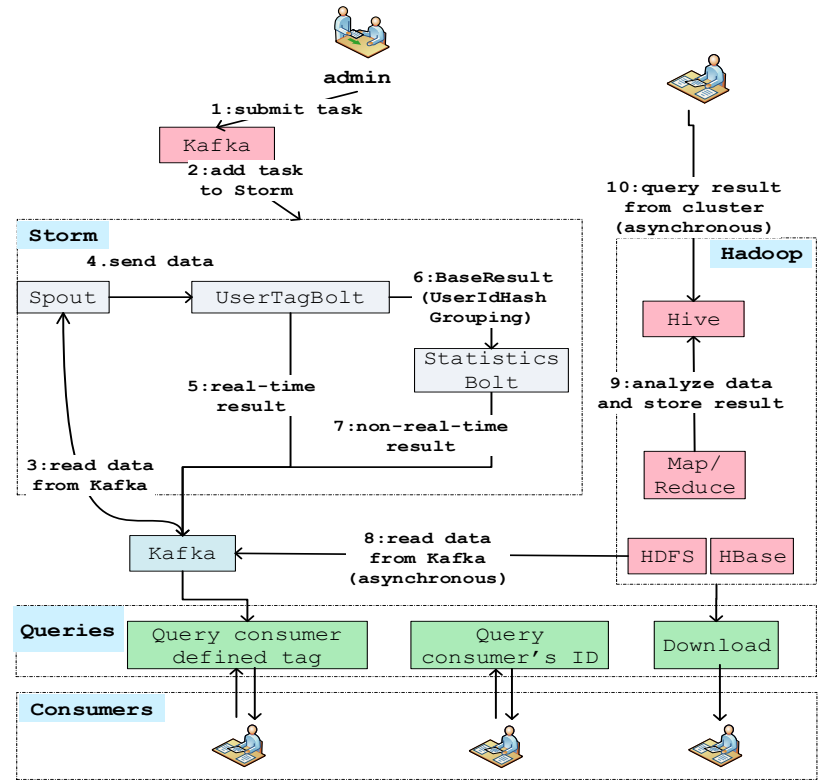

Figure 4. The elaborated DMP architecture.

Figure 5 illustrates the ETL operation within DMP, involving: (i) consumption of topics from Kafka; (ii) byte stream formatting with a relevant Avro schema; and (iii) Kafka messages' serialization with corresponding topics to HDFS.

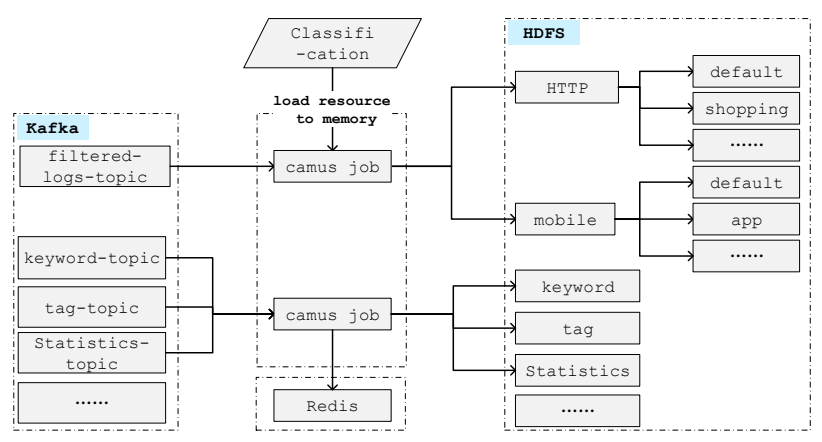

Figure 5. The ETL operation within DMP.

\footnotetext{
${ }^{4}$ https://kafka.apache.org/

${ }^{5} \mathrm{https} / / /$ hadoop.apache.org/docs/r1.2.1/hdfs design.html

${ }^{6} \mathrm{https}$ ://docs.confluent.io/1.0/camus/docs/intro.html
} 


\section{Modelling \& Simulation Tier of EMULSION}

The goal of using this tier is twofold: (i) to model CPS objects and IoT services, supplied by the EMULSION platform, along with their inherent attributes, and temporal/spatial/event characteristics. The Generalized Nets modelling [18] [19] is applied here to optimize the queuing disciplines used for serving the incoming requests for IoT services provided by EMULSION; (ii) to simulate the actual provision of IoT services as to determine the optimal platform configuration in each particular use case, by solving complex optimization tasks, as described in the next subsections.

\subsection{Determination of the optimal schemes for the deployment of sensors and/or monitoring stations}

This task aims at determining the optimal number of sensors and/or monitoring stations (in the sensor tier) needed to cover a particular area of interest as to minimize the capital expenditure (CAPEX) and operating expenditure (OPEX) while maintaining high key performance indicators' (KPIs) values. For instance, in the 'smart environment control' IoT domain, which is initially targeted by EMULSION, an important service relates to the continuous monitoring of the air quality index (AQI) in the target area(s) for the purposes of daily live reporting based on geo-grid positioned AQI monitoring stations, as shown in Figure 6.

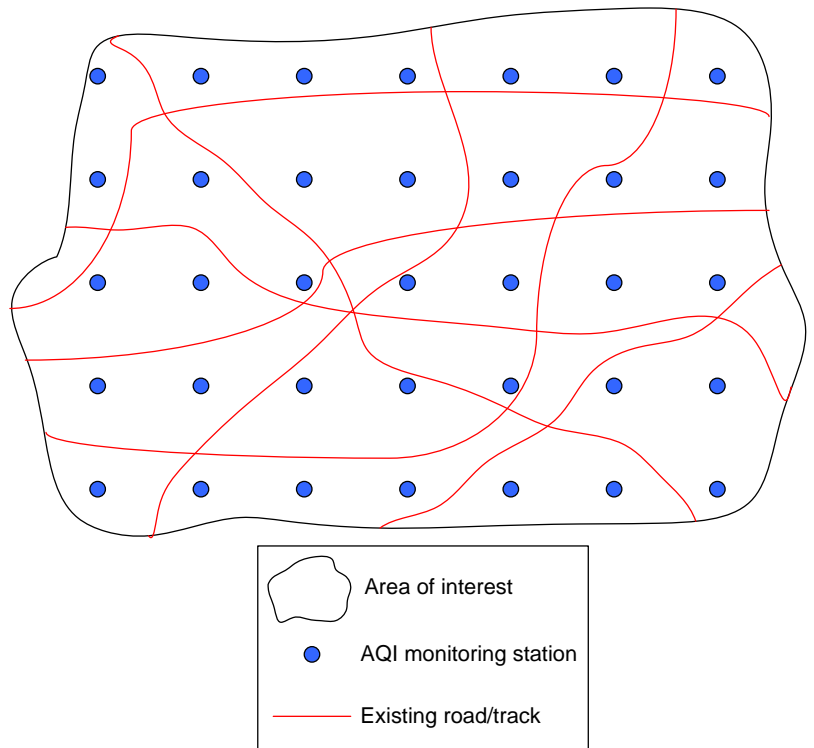

Figure 6. Using a geo-grid of AQI monitoring stations for covering a target area: the initial positioning of stations.

In addition, the AQI data obtained by such geogrid are used also for short/long-term forecasting of the future atmospheric environment conditions in the target area(s), for the purposes of smart proactive route planning, performed by the corresponding pilot prototype application, on behalf of consumers, as to find and recommend to them the 'healthiest' possible route to follow through that area(s).

However, taking into account that people in their movement, either on foot or by bike/vehicle, follow the tracks or roads existing in the area, an adjustment of the position of the AQI monitoring stations is required as to cover the existing track/road map, as shown in Figure 7.

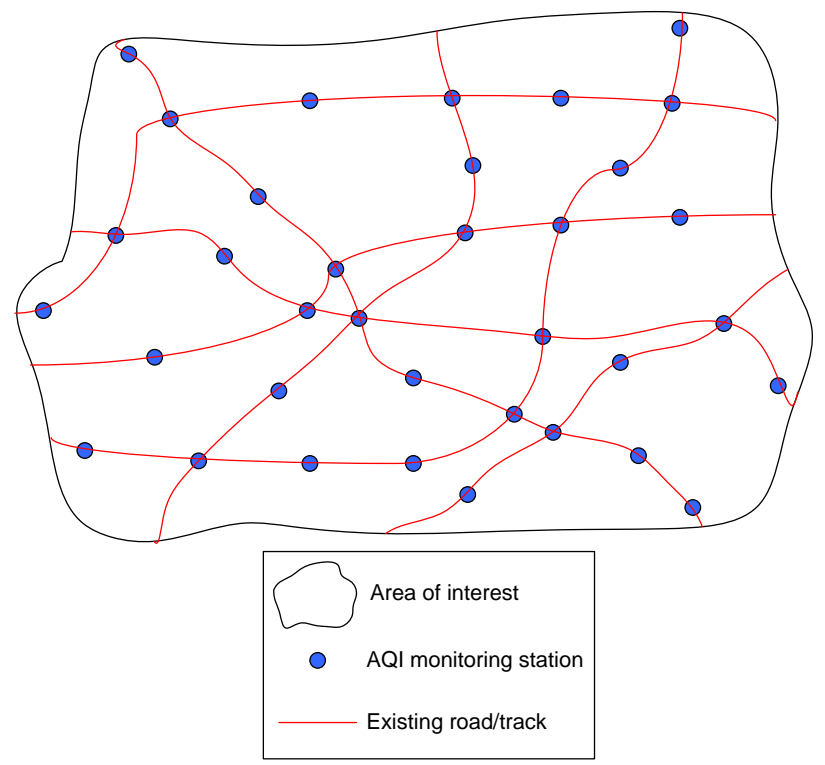

Figure 7. Using a geo-grid of AQI monitoring stations for covering a target area: the final positioning of stations after adjustment made by the EMULSION's modelling \& simulation tier by keeping the same number of stations in the area.

\subsection{Determination of the optimal schemes for the deployment of communication gateways}

This task aims at determining the optimal schemes for the deployment of (short-range) communication gateways (and associated fog nodes), given the actual/existing position of the sensors and/or monitoring stations already deployed in the sensor tier, as to minimize the CAPEX and OPEX while maintaining high KPI values of EMULSION. In the 'smart environment control' IoT domain, which is initially targeted by EMULSION, the key factor in this task is the maximum communication range supported by the corresponding wireless communication standard, utilized by the AQI monitoring stations. Figure 8 illustrates the solution for the sample use case presented in Figure 7. As can be seen from Figure 8 , just 5 short-range communication gateways (of a particular type) are 
needed to serve all monitoring stations deployed in the target area. In addition, it is advisable to allow the positioning of stations, if possible at all, in the serving area of not just one but multiple communication gateways for achieving higher reliability of operation. In other words, the AQI monitoring stations, located in the cross sections of the serving areas of two (or more) gateways, have the advantage of using secondary gateway(s) in the case of any fault, malfunctioning, or misconfiguration of the primary gateway they have been initially assigned to. The primary gateway in this case is determined in such a way as to achieve (almost) equal load for all gateways deployed in the area. This is shown in Figure 8, by using the same color for all monitoring stations assigned to a primary gateway of the same color. In this use case, each communication gateway gets equal load of serving 7 monitoring stations.

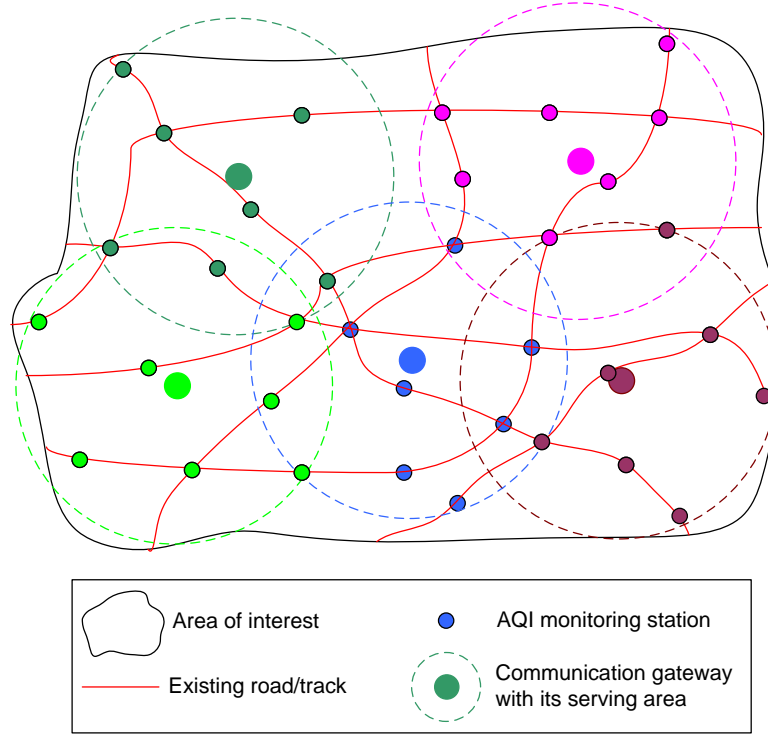

Figure 8. The optimal locations of the communication gateways, serving the AQI stations in a target area, as determined by the modelling \& simulation tier of EMULSION.

In addition, for easy access and management, it could be preferable to have a communication gateway mounted at the same point where a monitoring station is positioned. In this case, some additional adjustment of the communication gateways' locations might be needed as shown in Figure 9. However, this may lead to imposing a non-equal load to communication gateways. For instance, in the use case presented in Figure 9, the light-green colored gateway gets the least load (of 6 monitoring stations), whereas the blue-colored gateway gets the highest load (of 8 monitoring stations), compared to the remaining mid-loaded gateways (each serving 7 monitoring stations).

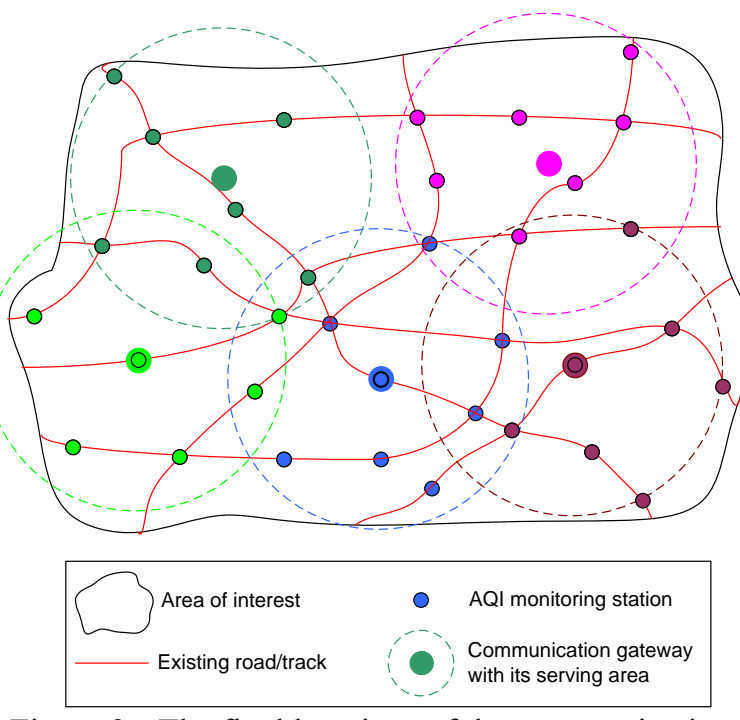

Figure 9. The final locations of the communication gateways, co-positioned with some of the AQI monitoring stations in the area.

\subsection{Determination of the optimal cloud configuration}

This task aims at determining the optimal configuration of the platform's cloud tier as to achieve an efficient provision of the corresponding IoT services. The results of performing this task with respect to determining the optimal (combined) configuration of the Kafka Brokers (KBs) and Storm Supervisors (SSs) in the cloud tier, are shown on Figure 10.

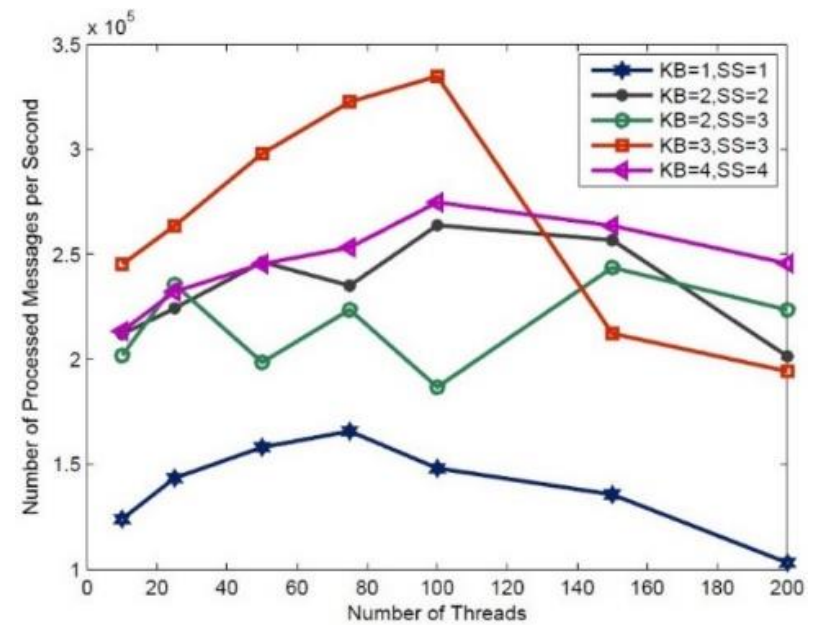

Figure 10. The number of messages per second, processed by different $\mathrm{KB} / \mathrm{SS}$ cloud configurations of EMULSION.

The presented results were obtained for the case of sending 10,000,000 messages from the sensor tier to the cloud tier, by utilizing different number of concurrency threads - starting with only 10 threads (with 1,000,000 messages processed by each thread) and ending up with 200 threads (with 50,000 
messages processed by each thread). In the case considered here, the most efficient operation of the platform was achieved by using a $3-\mathrm{KBs} / 3-\mathrm{SSs}$ cloud configuration, utilizing 100 threads (with 100,000 messages processed by each thread).

The presented results of the performance of the Kafka-Storm-Kafka part of the DMP core of the cloud tier clearly demonstrate that determining the $\mathrm{KB} / \mathrm{SS}$ appropriate numbers for use is of vital importance for achieving optimal operation of the EMULSION platform in real use case scenarios.

\section{Conclusion}

Elaborated as a typical example of the horizontal IoT platforms, the presented EMULSION IoT platform is able to meet the today's requirements for achieving multi-dimensional flexibility, scalability, interoperability, and easy adjustment to newemerging IoT scenarios and use cases. By considering the current consumer/service/network context, the designed platform is able to supply highly personalized, customized, and contextualized IoT services by utilizing distributed real-time 'big data' processing and analytical techniques in the cloud. This way, EMULSION can convert the collected sensing data and information about the IoT service activities of consumers into rich analytic datasets, which are utilized for the proactive realtime recommendation of the 'best' service instances for use by each individual consumer in conformance to the $A B C \& S$ communication paradigm [10]. While focusing primary on the 'smart environment control' and 'smart healthcare' IoT domains, EMULSION has the capacity for the supply of services in other IoT domains as well.

Specific attention in this paper has been paid to presenting some aspects of the modelling \& simulation tier of this platform, which is a novel architectural element, proposed here for inclusion in similar IoT platforms. The utilization of such tier allows to perform different optimization tasks in order to obtain the optimal platform configuration in each particular use case and thus achieve an efficient provision of IoT services and the best quality of experience (QoE) delivered to consumers anytime-anywhere-anyhow.

Future R\&D work will be focused on the elaboration of effective models of advanced IoT services for provision by EMULSION.

\section{References:}

[1]. Wopata, M., 5 Things to Know About the IoT Platforms Market. 2021, IoT Analytics.
[2]. Wegner, P., IoT Platform Companies Landscape 2021/2022: Market consolidation has started. 2021, IoT Analytics.

[3]. Lueth, K.L., IoT Platform Companies Landscape 2019/2020: 620 IoT Platforms globally. 2019, IoT Analytics.

[4]. Ganchev, I., Z. Ji, and M. O'Droma. A Generic Multi-Service Cloud-Based IoT Operational Platform - EMULSION. in 2019 International Conference on Control, Artificial Intelligence, Robotics \& Optimization (ICCAIRO). 2019.

[5]. Ganchev, I., Z. Ji, and M. O'Droma. A generic IoT architecture for smart cities. in Irish Signals \& Systems Conference 2014 and 2014 China-Ireland International Conference on Information and Communications Technologies (ISSC 2014/CIICT 2014). 25th IET. 2014. IET.

[6]. Yaici, K., IoT forecast: connectivity management platforms 2019-2028, in IoT Platforms and Technology. 2020.

[7]. Kontos, P., IoT platform enterprise contracts tracker $2 \mathrm{H}$ 2020, in IoT Platforms and Technology. 2021.

[8]. Ganchev, I., Z. Ji, and M. O'Droma, Designing a Low-Cost Location Tracker for Use in IoT Applications, in XXXIII General Assembly and Scientific Symposium of the International Union of Radio Science (URSI GASS 2020). 2020, IEEE. p. x1-x2.

[9]. Ganchev, I., Z. Ji, and O.D. M., Low-cost and ultra-low-power consuming RTUs for use in IoT systems. Int. J. Circ., Syst. and Sig. Process., 2020. 14: p. 76-82.

[10]. Ganchev, I., User-Centric and Context-Aware $A B C \& S$. International Journal "Information Technologies \& Knowledge", 2015. 9(3): p. 203-215.

[11]. Jing, E., et al., ECG Heartbeat Classification Based on an Improved ResNet-18 Model. Comput Math Methods Med, 2021. 2021: p. 6649970.

[12]. Wang, Y.F., et al., $R G$ Hyperparameter Optimization Approach for Improved Indirect Prediction of Blood Glucose Levels by Boosting Ensemble Learning. Electronics, 2021. 10(15): p. 1797.

[13]. Zhou, D., et al., Novel SDDM Rating Prediction Models for Recommendation Systems. IEEE Access, 2021. 9: p. 101197101206.

[14]. Zhang, H., et al., FeatureMF: An Item Feature Enriched Matrix Factorization Model for Item Recommendation. IEEE Access, 2021. 9: p. 65266-65276. 
[15]. Razouk, W., D. Sgandurra, and K. Sakurai, A new security middleware architecture based on fog computing and cloud to support IoT constrained devices, in Proceedings of the 1st International Conference on Internet of Things and Machine Learning. 2017, Association for Computing Machinery: Liverpool, United Kingdom. p. Article 35.

[16]. Lee, H., et al., A novel service-oriented platform for the internet of things, in Proceedings of the Seventh International Conference on the Internet of Things. 2017, Association for Computing Machinery: Linz, Austria. p. 1-8.

[17]. Perera, S. and S. Suhothayan. Solution patterns for realtime streaming analytics. in Proceedings of the 9th ACM International Conference on Distributed Event-Based Systems. 2015. ACM.

[18]. Andonov, V., S. Poryazov, and E. Saranova, Generalized net representations of control structures in service systems theory. Advanced Studies in Contemporary Mathematics (Kyungshang), 2020. 30(1): p. 49-60.
19]. Andonov, V., S. Poryazov, and E. Saranova, Generalized net representations of elements of service systems theory. Advanced Studies in Contemporary Mathematics (Kyungshang), 2019. 29(2): p. 179-189.

Ivan Ganchev carried out the conceptualization, methodology, project administration and supervision, funding acquisition, writing of the original draft and editing of the final paper.

Zhanlin Ji carried out data curation, formal analysis, investigation, validation, software development, and resource management.

This publication has emanated from joint research conducted with the financial support of the Bulgarian National Science Fund (BNSF) under the Grant No. KP-06-IP-CHINA/1 (КП06-ИП-КИТАЙ/1) and the S\&Т Major Project of the Science and Technology Ministry of China, Grant No. 2017YFE0135700.

\section{Creative Commons Attribution License 4.0 (Attribution 4.0 International, CC BY 4.0)}

This article is published under the terms of the Creative Commons Attribution License 4.0

https://creativecommons.org/licenses/by/4.0/deed.en US 